\title{
Extracting Implicit Programming Rules: Comparing Static and Dynamic Approaches
}

\author{
Tarannum Shaila Zaman \\ Department of Computer Science \\ University of Kentucky \\ Lexington, Kentucky, USA \\ tarannum.zaman@uky.edu
}

\author{
Tingting Yu \\ Department of Computer Science \\ University of Kentucky \\ Lexington, Kentucky, USA \\ tyu@cs.uky.edu
}

\begin{abstract}
Programs often follow implicit programming rules, such as, function call $A$ must be followed by function call $B$. Rules of such kinds are rarely documented by developers. Nevertheless, programming rules play an important role in software testing and maintenance. For example, the rules can be used as test oracles to detect violations. If a programmer can be notified of these rules before updating the source code, the chances of generating defects due to rule violations might be minimized. Prior works have used static and dynamic analysis techniques to extract implicit programming rules, but none compares the effectiveness of the two techniques. In this paper, we have undertaken an empirical study to compare the two techniques when they are being used for extracting programming rules. Our results indicate that the performance of the dynamic analysis technique depends on the number and the diversity of the traces. Moreover, the dynamic analysis technique generates more precise rules than the static analysis technique if a diverse and sufficient number of test cases are provided.
\end{abstract}

\section{CCS CONCEPTS}

- Information systems $\rightarrow$ Association rules; • Software and its engineering $\rightarrow$ Software testing and debugging;

\section{KEYWORDS}

Implicit Programming Rules, Static Analysis, Dynamic analysis, Empirical study

\section{ACM Reference Format:}

Tarannum Shaila Zaman and Tingting Yu. 2018. Extracting Implicit Programming Rules: Comparing Static and Dynamic Approaches. In Proceedings of the 7th International Workshop on Software Mining (SoftwareMining '18), September 3, 2018, Montpellier, France. ACM, New York, NY, USA, 7 pages. https://doi.org/10.1145/3242887.3242889

\section{INTRODUCTION}

Implicit programming rules can be useful in many ways. One usage scenario is 'test oracle' generation. A quality test oracle can be very helpful in detecting bugs. The rules can also be helpful to software

Permission to make digital or hard copies of all or part of this work for personal or classroom use is granted without fee provided that copies are not made or distributed for profit or commercial advantage and that copies bear this notice and the full citation on the first page. Copyrights for components of this work owned by others than ACM must be honored. Abstracting with credit is permitted. To copy otherwise, or republish, to post on servers or to redistribute to lists, requires prior specific permission and/or a fee. Request permissions from permissions@acm.org.

SoftwareMining '18, September 3, 2018, Montpellier, France

(c) 2018 Association for Computing Machinery.

ACM ISBN 978-1-4503-5975-7/18/09 . \$ \$15.00

https://doi.org/10.1145/3242887.3242889 developers. In industry, the source code size of some software can be huge. Most of the time no documentation is maintained for the implicit programming rules. Hence, it becomes very challenging for a developer to update the source code or locate the defects. Moreover, some rules can change in the new versions of the software or the number of rules can be increased or decreased with the change in the source code. As a result, no document is maintained for the software rules. Violations of these rules are common for programmers that can introduce bugs, especially for new programmers who are unaware of these rules. [17].

There are many implicit programming rules in a large software - some are simple and some are complex. For example, the function call pair of lock and unlock is a simple programming rule. A complex rule can involve a list of function call and system call sequence. Data mining techniques have been used to extract implicit programming rules [26] from either source code [17] [5] or dynamic execution traces [8] [4]. In this paper, we perform an empirical study to decide which type of programming analysis can be more advantageous to extract implicit programming rules. Some comparisons are also shown for the dynamic analysis portion by varying the number of run-time traces.

There has been some existing work on extracting implicit programming rules. For example, PR-Miner [17] extracts programming rules in general forms by analyzing the source code of a large software. Another technique [5] conducted by Engler et. al extracts only pair-wise programming rules like function $A$ must be paired with function $B$. Static analysis does not need to execute the program and is often fast and able to reason about the entire program. However, one disadvantage of static analysis is that it may report false negatives (i.e., not able to reason all possible paths). For example, the main limitation of PR-Miner [17] is that it generates false negative rules in detecting violation. However, PR-Miner is very fast in generating closed programming rules(e.g., takes only 1-42 seconds to extract more than 32,000 rules).

There has also been some work on using dynamic analysis techniques to mine execution traces for extracting programming rules [8] [4]. The dynamic analysis does not require the availability of source code and is precise because it relies on actual executions. The main disadvantage of dynamic analysis is that it may miss programming rules that are not exercised by inputs (i.e., false-negatives) and also when one input is exercised multiple times, it may generate some rules which are not frequent rules (i.e., false-positives).

The main objective of our work is to conduct an empirical study to compare static and dynamic analysis approaches in generating implicit programming rules. In this paper, we implement two techniques: one based on static analysis and the other based on dynamic 
main ()$\rightarrow$ set program name ()

main(), set_program_name ()$\rightarrow$ close_stream(), close_stdout () main(),GEAcompile() $\rightarrow$

set_program_name(), xmalloc(), close_stream(), close_stdout()

Figure 1: Three function call rules collected from grep program

$$
\begin{gathered}
\text { main }() \rightarrow \text { do_stat }() \\
\text { main }(), \text { do_stat }()() \rightarrow \text { _do_global_dtors_aux }()
\end{gathered}
$$

Figure 2: Two Function call rules collected from gzip program

analysis. For static technique, we analyze the source code of a program to enumerate program paths and then apply data mining techniques to generate programming rules from those paths. Our static analysis rule generation technique is inspired by the PR-Miner [17] technique. However, we focus on rules involving function calls and system calls, whereas PR-Miner focuses on rules involving functions, variables, and data types. For dynamic analysis, we collect runtime traces by executing the program against its test cases. We then apply data mining techniques to generate programming rules from the runtime traces. There is no prior work on generating implicit rules by using the dynamic analysis technique. However, our dynamic analysis approach is inspired by some previous work [8] [4], which mines execution traces for collecting certain runtime information.

Finally, we conduct an empirical analysis to compare the performance of the two techniques. The results show that, when we have a diverse and sufficient number of test cases to generate runtime traces, the dynamic approach generates more rules than the static approach. Additionally, the rules generated by the dynamic approach are more precise than those generated by the static approach.

This paper is organized as follows. The motivation of our work is presented in section 2. Section 3 describes some background information about the study. The evaluation details is described in section 4 . Section 5 depicts results of the experiments. The related work is discussed in section 6 and finally, section 7 concludes the paper stating some future directions.

\section{MOTIVATION}

Writing programs often follows certain general patterns. One common example in a program is a system call pair: open and close. An open system call should be followed by a close system call. This is a common programming rule. However, every program has some implicit programming rules and those rules are not so simple. In case of large programs, to find out those rules are really very tough and most of the time the programmers do not have a document for these rules. If we can provide the developers a way to generate implicit programming rules automatically, it will be very helpful for them. That means, the rules we generate automatically can not only be useful in the testing phase but also in the developing phase.
The Figure 1 and Figure 2 show several programming rules in grep [19] and gzip [18] programs, respectively. These rules are related to the function calls of the programs. A function is an item and an itemset contains one or more items. The right arrow represents the correlation between the two itemsets in a rule. The first rule implies that when the grep program starts with the main function, it must call the set_program_name function to continue the program. Likewise, the third rule means that when the GEAcompile function and the main function happens together, the program shall call the set_program_name, xmalloc, close_stream and close_stdout functions.

In this paper, we study the static and dynamic analysis techniques to extract programming rules. For static analysis, we enumerate all paths of the program with a certain loop boundary. For dynamic analysis, we collect execution traces obtained by running test cases. We then use data mining algorithm [9] [12] to extract programming rules from the traces and paths. We conduct an empirical study on those rules collected by both dynamic and static analysis to see which technique performs better. Table 1 represents the program paths collected by static analysis (Column 1) and the execution traces collected at the run-time (Column 2) for the Linux coreutils program $m v$. These paths and traces are used to generate programming rules involving system calls. To better understand the techniques, in this table, we keep the number of paths and traces the same.

When we apply the Fp-Close association rule mining algorithm [9] on the data from the column 1 of Table 1, we get 6 association rules. For the data shown in column 2, we get 14 association rules. The results show that more rules are collected by the dynamic technique. This happens because paths obtained from simple static analysis are often imprecise. It can both generate unfeasible paths and miss important paths. In that case, some interesting rules may not be captured by static analysis. For example, the rule unlink $\rightarrow$ rename is generated from the dynamic traces but is not generated from the static paths. This rule is a very interesting rule and violation of this rule can cause a race condition bug [23].

However, while dynamic analysis can extract more rules, it may also generate a number of false positive and false negative rules. This is because, the rules generated by dynamic analysis completely depend on the traces collected from different inputs.

\section{BACKGROUND}

\subsection{Frequent Pattern Mining}

Frequent patterns are item-sets (i.e., sets of items) that appear in a dataset with the frequency no less than a user-specified threshold [11](i.e., support value). For example, Table 2 shows five transactions, where each transaction corresponds to an execution trace consisting of function names (i.e., items). If the minimum support value (i.e., minsup) is set to $80 \%$, we will get frequent patterns: $\{\mathrm{F} 5\}$, $\{\mathrm{F} 2, \mathrm{~F} 5\},\{\mathrm{F} 3\}$ and $\{\mathrm{F} 2\}$. To make the patterns precise, the value of minsup should be kept high.

Over the years several algorithms [3] [12] [27] are developed to mine frequent patterns. In this work, we used the Fp-Growth [12] algorithm to mine frequent patterns. Fp-Growth algorithm works by constructing Fp-tree and performance studies shows that this method is very efficient [11]. 
Table 1: Applying Two Techniques on $m v$ program

\begin{tabular}{|l|l|}
\hline Paths Collected by Static Analysis & Traces Collected by Dynamic Analysis \\
\hline 1. $\{$ stat, lstat, access, rename $\}$ & 1. $\{$ stat, lstat, lstat, access, unlink, rename $\}$ \\
\hline 2. $\{$ stat, lstat, access, unlink, rename $\}$ & 2. $\{$ stat, lstat, lstat, access, unlink, rename $\}$ \\
\hline 3. $\{$ stat, lstat, access, rename $\}$ & 3. $\{$ stat, lstat, lstat, access, unlink, open, close, rename $\}$ \\
\hline 4. $\{$ stat, lstat, rename $\}$ & 4. $\{$ stat, lstat, lstat, rename $\}$ \\
\hline
\end{tabular}

Table 2: A transaction Database

\begin{tabular}{|c|c|}
\hline Transaction no. & Item Names \\
\hline T1 & F1, F3, F4 \\
\hline T2 & F2, F3, F5 \\
\hline T3 & F1, F2, F3, F5 \\
\hline T4 & F2, F5 \\
\hline T5 & F1, F2, F3, F5 \\
\hline
\end{tabular}

\subsection{Closed Frequent Pattern Mining}

Closed frequent patterns are the patterns, where none of its supersets has the same support as the itemset [28]. We can illustrate this definition by the given example of table 2. When we run Fp-close [9] algorithm with a minimum support $80 \%$ on the dataset of table 2, we get two closed frequent patterns: $\{\mathrm{F} 2, \mathrm{~F} 5\},\{\mathrm{F} 3\}$. As the results show, the closed frequent patterns do not contain $\{\mathrm{F} 5\}$ or $\{\mathrm{F} 2\}$. The reason is that, the two patterns have the same support and are the superset of pattern $\{\mathrm{F} 2, \mathrm{~F} 5\}$.

\subsection{Association Rule Mining}

An association rule is an implication expression of the form $\mathrm{X} \rightarrow \mathrm{Y}$, where $\mathrm{X}$ and $\mathrm{Y}$ are disjoint item-sets. Support and Confidence are two terms that are used to measure the strength of the association rules. A rule, which has a very low support value may occur by chance. Hence, those rules are uninteresting. Confidence is important because it measures the reliability of the inference made by a rule [24].

Support (S): Fraction of transactions that contain both $\mathrm{X}$ and $\mathrm{Y}$.

$\mathrm{S}=($ number of transactions that contain $\mathrm{X}, \mathrm{Y}) /$ number of all transactions

Confidence(C): Measures how often items in Y appear in transactions that contain $\mathrm{X}$.

$\mathrm{C}=($ number of transactions that contain $\mathrm{X}, \mathrm{Y}) /$ number of transactions that contain $\mathrm{Y}$.

In this work, the data mining algorithm Fp-Growth association rule mining [12] is used to find the implicit programming rules and Fp-Close association rule mining algorithm is used to find the closed programming rules[9]. Association rule generation by using the FP-Growth algorithm often consists of two steps. First, finding out the frequent itemset for a given minimum support. Second, forming the rules by using the frequent itemsets and the minimum confidence constraint [1]. If we demonstrate the algorithm on the dataset of the table 2 where minimum support is $80 \%$ and the minimum confidence $80 \%$, then we get these rules:
$\mathrm{F} 5 \rightarrow \mathrm{F} 2 ; \mathrm{S}=80 \%$ and $\mathrm{C}=100 \%$

$\mathrm{F} 2 \rightarrow \mathrm{F} 5 ; \mathrm{S}=80 \%$ and $\mathrm{C}=100 \%$

\section{EVALUATION}

In this study, we consider three research questions.

- RQ1: Which analysis technique generates more number of implicit programming rules?

- RQ2: Which analysis technique is more precise?

- RQ3: How does variation of the number of traces affect the number of programming rules generated?

The first research question compares the number of rules generated by static and dynamic approaches. The second question evaluates the precision of the two approaches in generating programming rules. Lastly, the third one examines whether the number of generated implicit rules will remain the same as the number of traces increases or decreases.

\subsection{Subjects and Implementation}

We use three subject programs grep, gzip, and mv, among them the first two programs are collected from the Software-artifact infrastructure repository (SIR) of University of Nebraska-Lincoln [2] and the mv program is a open source program. We selected these programs because their test cases are available, which allows us to easily study the dynamic approach. grep contains 169 test cases, gzip contains 214 test cases, mv contains 79 test cases. For answering RQ1 and RQ2, we conduct our experiments on subject program grep and mv and for answering RQ3, we use all the three subjects. Table 3 shows the three programs with the total number of lines and characteristics.

Table 3: Characteristics of subjects used in this evaluation

\begin{tabular}{|c|c|c|}
\hline Program & LOC & Characteristics \\
\hline grep & 6905 & Search and print regular expression. \\
\hline gzip & 7252 & Data compression program. \\
\hline mv & 7002 & Moves one or more files or directories. \\
\hline
\end{tabular}


Table 4: Experimental study to observe the behavior of three different programs by changing the number of traces

\begin{tabular}{|l|l|l|l|l|l|l|l|}
\hline Prog_name & Traces & F_Items & Cls_F_Items & Rules & Precision_rules & Clsrules & Precision_clsrules \\
\hline grep & 168 & 63 & 3 & 602 & $58.57 \%$ & 106 & $67.94 \%$ \\
\hline grep & 148 & 63 & 3 & 602 & $58.47 \%$ & 106 & $67.94 \%$ \\
\hline grep & 114 & 15 & 1 & 50 & $61.23 \%$ & 14 & $71.4 \%$ \\
\hline grep & 71 & 15 & 1 & 50 & $61.23 \%$ & 14 & $71.4 \%$ \\
\hline grep & 31 & 31 & 2 & 188 & $54.26 \%$ & 2 & $100 \%$ \\
\hline gzip & 214 & 511 & 6 & 1866 & $51.7 \%$ & 99 & $59.60 \%$ \\
\hline gzip & 157 & 151 & 5 & 611 & $65.47 \%$ & 94 & $53.19 \%$ \\
\hline gzip & 100 & 31 & 2 & 180 & $66.67 \%$ & 44 & $68.18 \%$ \\
\hline gzip & 64 & 31 & 1 & 180 & $66.67 \%$ & 30 & $73.33 \%$ \\
\hline gzip & 19 & 31 & 1 & 180 & $66.67 \%$ & 30 & $73.33 \%$ \\
\hline mv & 102 & 96 & 17 & 669 & $54.86 \%$ & 71 & $64.79 \%$ \\
\hline mv & 68 & 47 & 7 & 430 & $53.72 \%$ & 41 & $68.29 \%$ \\
\hline mv & 59 & 29 & 6 & 264 & $66.29 \%$ & 34 & $67.65 \%$ \\
\hline mv & 16 & 3 & 1 & 2 & $100 \%$ & 2 & $100 \%$ \\
\hline
\end{tabular}

We implement the static analysis using Codesurfer [10], a static analysis framework. We first construct the inter-procedural control flow graph (CFG) for the program and then use the depth-first search algorithm with a constant loop boundary to enumerate all paths. For the dynamic technique, we use PIN [13], a binary instrumentation tool to collect traces for different test cases.

Both types of traces contain only function calls. From those traces, hash values are generated and all the traces that contain the hash values of the function names are saved in a single file for each program and for each analysis approach. This file is used as the input file for the data mining algorithm to generate association rules. SPMF [7], an open-source data mining âĂŞlibrary is used to apply both Fp-Growth [12] and Fp-Close[9] algorithms. In both cases, the minimum support and the minimum confidence values are set to $90 \%$.

\subsection{Metrics}

After generating implicit rules for the two techniques, we calculate the precision of those rules. Precision represents the percentage of the real programming rules from the rules generated by our technique. For calculating the precision we use the following formula [15]:

\section{Precision $=($ true_positive $) /($ true_positive + false_positive $)$}

To determine whether a rule is a false positive, we manually examine the code. If the rule does not hold in at least one path, it is marked as false positive.

\subsection{Threats to Validity}

4.3.1 Threats to Internal Validity. For collecting the dynamic traces the programs have to run with various test cases. The result of the dynamic analysis phase largely depends on the test cases. Hence, the primary threat to internal validity are the biases in the test cases. To reduce this threat, two programs are used in this study for which, test cases are already generated by other programmers. One open source program is used whose test cases are available on the web. In this study, we, the authors, had to judge our own work.
However, the two techniques presented here, are developed by us. Hence, there is a little chance that we are biased to a particular technique. Overall, for reducing these biases, an empirical analysis is done over the two techniques.

4.3.2 Threats to External Validity. The primary threat to external validity is to answer the question: what if the results maintain same type of behavior for multiple programs. To reduce this threat to validity, one experiment is conducted over two programs and the other is conducted over three programs. In future, more subjects will be observed with these techniques.

4.3.3 Threats to Conclusion Validity. To reduce the conclusion validity of this work, the experiment is repeatedly performed by keeping the same setup and inputs. The measuring method for this study is also justified in the previous section.

4.3.4 Threats to Construct Validity. The main construct validity of this work is the Mono operation bias. To reduce this bias, we conducted one of our experiment on two projects and the other one over three projects. We believe our study properly represents the construct, as the programs are real-world programs and are not very small.

\section{RESULTS AND ANALYSIS}

\subsection{RQ1: Comparing the Number of Rules Generated by Static and Dynamic Approaches}

For answering this research question, we have done experiments of two subjects. Table 5 shows the results of the programs grep and $m v$. Columns 1-2 display the analysis techniques and the program names. Columns 3-5 report the number of generated paths/traces, the number of frequent items, and the number of rules. The results are further plotted in figure 5 and figure 6 . The results show that more implicit programming rules are generated from dynamic traces. 


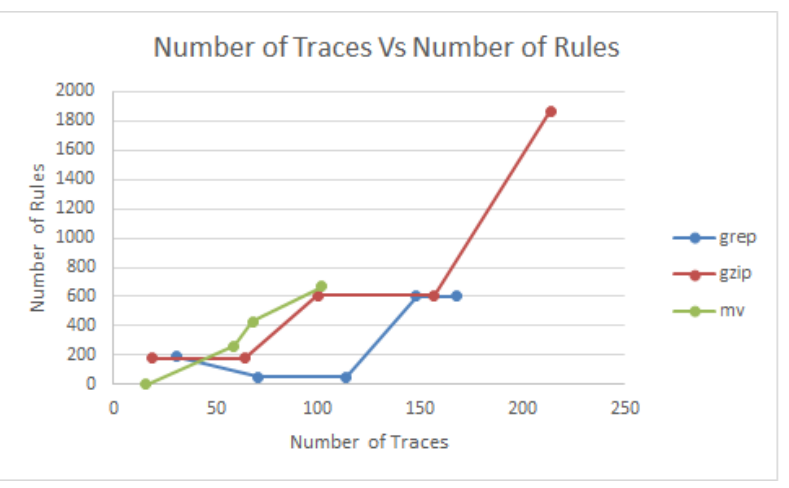

Figure 3: Number of traces Vs Number of generated rules

Table 5: The Number of generated rules and precision scores from dynamic and static analysis approaches

\begin{tabular}{|c|c|c|c|c|c|}
\hline Analysis & Prog & Paths/Traces & F_items & Rules & Precision \\
\hline Dynamic & grep & 169 & 63 & 602 & $58.57 \%$ \\
\hline Static & grep & 202 & 47 & 218 & $55.04 \%$ \\
\hline Dynamic & mv & 102 & 96 & 669 & $54.86 \%$ \\
\hline Static & mv & 69 & 31 & 213 & $49.77 \%$ \\
\hline
\end{tabular}

\subsection{RQ2: Comparing the Precision between Static and Dynamic Approaches}

Column 6 of Table 5 reports the precision scores of the two approaches for the two programs. The results indicate that the dynamic approach outperforms the static approach in terms of precision.

\subsection{RQ3: Varying the Number of Traces in Dynamic Approach}

We vary the number of traces generated by the dynamic approach on the three programs. Table 4 shows the results. Columns 1- 5 reports the name of the program, the number of traces, the number of frequent item-sets, the number of closed frequent item-sets, and the number of generated implicit programming rules, the precision of those rules, the number of closed implicit programming rules, and the precision of those rules.

The results indicate that on $g z i p$ and $m v$, when the number of traces increases then generally more rules are generated. However, on grep program, the number of generated rules do not always increase with the number of traces. It shows that the number of rules remain same or increase with the increasing number of traces. Figure 3 shows the graphical representation of the results. These results indicate that the number of generated rules and precision totally depend on the number and variety of test cases as well as traces.

\subsection{Discussion}

In our experiment, we used two data mining algorithm to generate rules: Fp-growth [9] and Fp-close [12]. However, Fp-close in most cases generates less number of rules than Fp-growth, as we know,

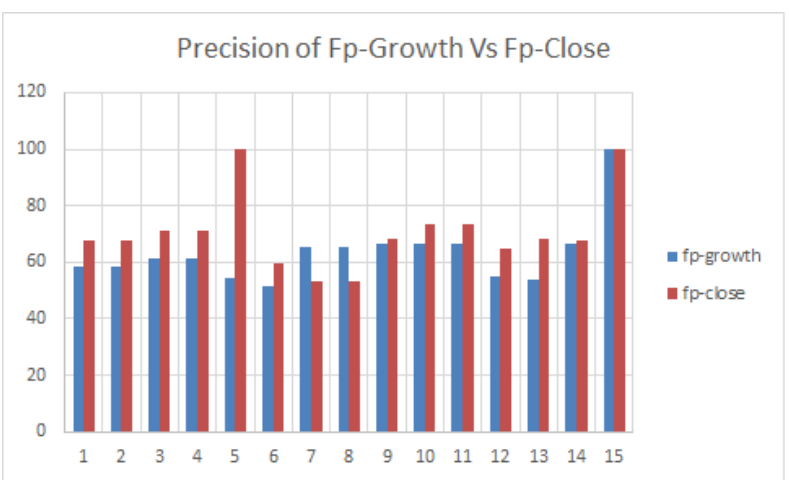

Figure 4: Precision of generated rules in Fp-growth and in Fp-Close

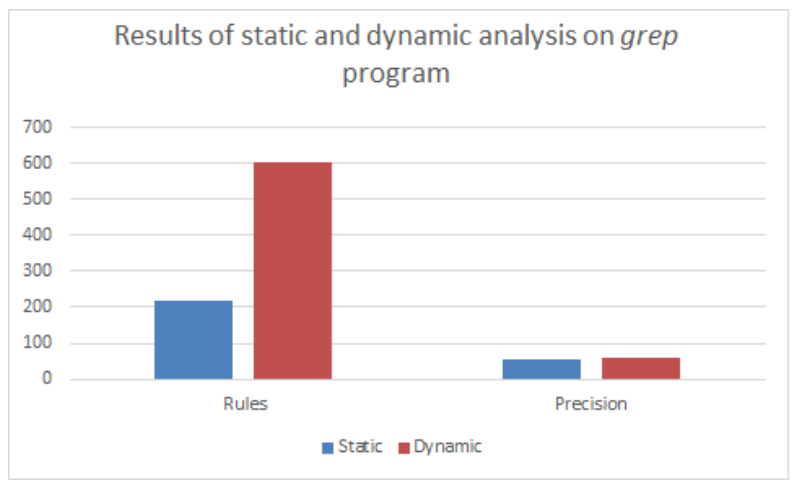

Figure 5: Comparison of number of generated rules and their precision from static and dynamic analysis for grep program.

the rules generated by Fp-close actually are the superset of all rules of the Fp-growth. For this reason, we did not do any comparison between the number of rules generated by these two techniques. However, Figure 4 shows a comparison between the precision of rules generated by the Fp-growth algorithm and the precision of the rules generated by the Fp-close algorithm. The figure shows that, on an average, the percentage of precision of the rules generated by the Fp-close is higher than the rules generated by the Fp-growth.

Overall, the results from our study imply that dynamic analysis always outperforms the static analysis in terms of the number and the precision of the rules. The results of dynamic analysis mostly depend on the number and types of test cases. If a large and diverse number of traces can be collected, dynamic analysis generates more implicit programming rules.

\section{RELATED WORKS}

Li et al. proposed a method called PR-Miner [17] which uses a data mining technique known as frequent itemset mining to efficiently extract implicit programming rules from large software code written in an industrial programming language such as $\mathrm{C}$. In their work, the authors use the static analysis of the source code to find the rules. They parse the source code and build an itemset database. 


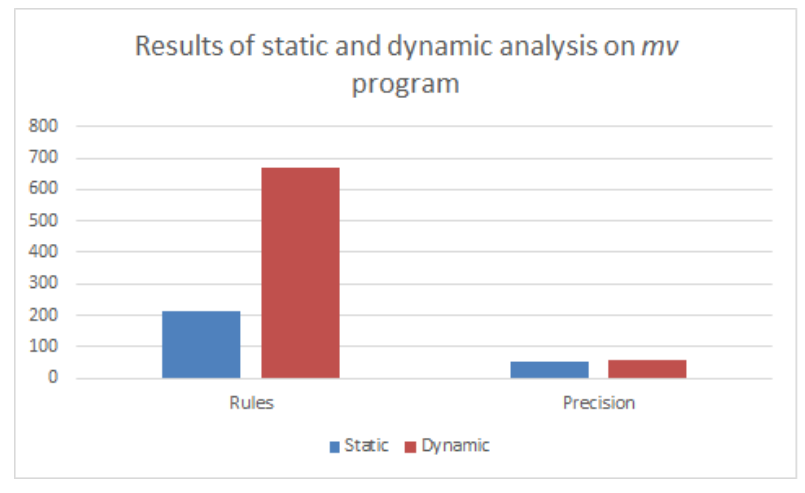

Figure 6: Comparison of number of generated rules from static and dynamic analysis for $m v$ program

Thus, they convert the programming pattern extraction problem into a frequent itemset mining problem. In our work, we examine the source code flow in the static phase as well as in the dynamic phase. In the case of static analysis, if the minimum support is kept too high, many interesting patterns might not be identified. Hence, a dynamic analysis can provide a chance to observe how it works in rules' generation process. This work provides an empirical analysis where the number of generated rules is compared for dynamic and static analysis by keeping the minimum support and minimum confidence level same.

Li et al. proposed a technique named CP-Miner [16] where they use data mining techniques to identify copy-pasted code in large software suites and detect copy-paste bugs. They also discover the repeated patterns of the codes. However, in our work, we not only examine the copy paste items but also examine few other frequent patterns.

CAR-Miner [25] is an approach that mines exception handling rules. This technique also uses the static analysis of the source code. The authors construct Exception-Flow Graphs (EFG), which are an extended form of Control-Flow Graphs (CFG) and mine association rules from the graphs. In our work, we find the general implicit rules for function call and system call sequences by using both static and dynamic analysis of source code.

Some prior works attempt to summarize the rule mining techniques. Khatoon et al. [14] represent comparison and evaluation of the current state-of-the-art in source code mining techniques. The main objective of their work is to pinpoint the strong and weak points of the current tools and techniques. However, they did not provide any empirical study. In contrast, we implement two techniques and provide an empirical study to compare their performance.

Many other techniques are also proposed for generating automated test oracles. Dynamic invariant detection can extract specifications from programs dynamic executions [6] [22]. Nimmer and Ernst investigate the relationship between dynamic and static information and show that dynamic specification generation can capture some nontrivial and useful semantic information [20] [21].

\section{CONCLUSION AND FUTURE DIRECTION}

We have implemented two techniques for generating programming rules by using the association rule mining algorithm where the probability of generating false-positive rules is controlled by keeping the minimum support and minimum confidence value high. In future, we plan to develop one automated system to check the validity of the rules; so that, the minimum support can be kept average, and that will help reduce the percentage of false negative rules. We have investigated two programs to conduct this study. More programs can be studied with these techniques for getting more convincing results. The result of this study largely depends on the test cases. If test cases from other sources could be used in this study, the result would have been different. Hence, the good results of the dynamic analysis phase are completely dependent on the number and variety of test cases. In this work, the test cases and source code are collected from the SIR dataset. In future, one automated test case generation tool can be used to generate test cases, which will cover all the paths. Our study indicates that the dynamic analysis technique performs better in generating implicit programming rules over the static analysis of source code if a good number and varieties of traces are present. In the dynamic analysis, the number of generated rules completely depends on the number of traces. It is quite evident from our analysis that if a complete test case document is available for a source code, then dynamic analysis of source code can give good results than the static analysis. In future, more experiments can be performed on various types of software to support these results.

\section{ACKNOWLEDGMENTS}

This research is supported in part by the NSF grant CCF-1652149.

\section{REFERENCES}

[1] $[\mathrm{n} . \mathrm{d}$.$] Mining Frequent Itemsets - Apriori Algorithm.$ http://software.ucv.ro/ cmihaescu/ro/teaching/AIR/docs/Lab8-Apriori.pdf.

[2] $[\mathrm{n} . \mathrm{d}$.]. Software-artifact Infrastructure Repository. http://sir.unl.edu/php/previewfiles.php.

[3] Rakesh Agrawal, Tomasz Imieliński, and Arun Swami. 1993. Mining Association Rules Between Sets of Items in Large Databases. SIGMOD Rec. 22, 2 (June 1993), 207-216. https://doi.org/10.1145/170036.170072

[4] Christoph Csallner and Yannis Smaragdakis. 2006. Dynamically Discovering Likely Interface Invariants. In Proceedings of the 28th International Conference on Software Engineering (ICSE '06). ACM, New York, NY, USA, 861-864. https: //doi.org/10.1145/1134285.1134435

[5] Dawson Engler, David Yu Chen, Seth Hallem, Andy Chou, and Benjamin Chelf. 2001. Bugs As Deviant Behavior: A General Approach to Inferring Errors in Systems Code. SIGOPS Oper. Syst. Rev. 35, 5 (Oct. 2001), 57-72. https://doi.org/ 10.1145/502059.502041

[6] Michael D. Ernst, Jake Cockrell, William G. Griswold, and David Notkin. 1999. Dynamically Discovering Likely Program Invariants to Support Program Evolution. In Proceedings of the 21st International Conference on Software Engineering (ICSE '99). ACM, New York, NY, USA, 213-224. https://doi.org/10.1145/302405.302467

[7] Philippe Fournier-Viger, Jerry Chun-Wei Lin, Antonio Gomariz, Ted Gueniche, Azadeh Soltani, Zhihong Deng, and Hoang Thanh Lam. 2016. The SPMF OpenSource Data Mining Library Version 2. In Machine Learning and Knowledge Discovery in Databases, Bettina Berendt, Björn Bringmann, Élisa Fromont, Gemma Garriga, Pauli Miettinen, Nikolaj Tatti, and Volker Tresp (Eds.). Springer International Publishing, Cham, 36-40.

[8] Mark Gabel and Zhendong Su. 2008. Javert: Fully Automatic Mining of General Temporal Properties from Dynamic Traces. In Proceedings of the 16th ACM SIGSOFT International Symposium on Foundations of Software Engineering (SIGSOFT '08/FSE-16). ACM, New York, NY, USA, 339-349. https://doi.org/10.1145/1453101. 1453150

[9] Gosta Grahne and Jianfei Zhu. 2005. Fast Algorithms for Frequent Itemset Mining Using FP-Trees. IEEE Trans. on Knowl. and Data Eng. 17, 10 (Oct. 2005), 1347-1362. https://doi.org/10.1109/TKDE.2005.166 
[10] GrammaTech. [n. d.]. CodeSurfer. www.grammatech.com/products/codesurfer.

[11] Jiawei Han, Hong Cheng, Dong Xin, and Xifeng Yan. 2007. Frequent pattern min ing: current status and future directions. Data Mining and Knowledge Discovery 15, 1 (01 Aug 2007), 55-86. https://doi.org/10.1007/s10618-006-0059-1

[12] Jiawei Han, Jian Pei, and Yiwen Yin. 2000. Mining Frequent Patterns Without Candidate Generation. SIGMOD Rec. 29, 2 (May 2000), 1-12. https://doi.org/10. $1145 / 335191.335372$

[13] Osnat Levi (Intel). 2012. Pin - A Dynamic Binary Instrumentation Tool. https://software.intel.com/en-us/articles/pin-a-dynamic-binaryinstrumentation-tool.

[14] Shaheen Khatoon, Guohui Li, and Azhar Mahmood. 2013. Comparison and Evaluation of Source Code Mining Tools and Techniques: A Qualitative Approach Intell. Data Anal. 17, 3 (May 2013), 459-484. https://doi.org/10.3233/IDA-130589

[15] William Koehrsen. 2018. Beyond Accuracy: Precision and Recall https://towardsdatascience.com/beyond-accuracy-precision-and-recall3da06bea9f6c.

[16] Zhenmin Li, Shan Lu, Suvda Myagmar, and Yuanyuan Zhou. 2004. CP-Miner A Tool for Finding Copy-paste and Related Bugs in Operating System Code. In Proceedings of the 6th Conference on Symposium on Opearting Systems Design \& Implementation - Volume 6 (OSDI'04). USENIX Association, Berkeley, CA, USA, 20-20. http://dl.acm.org/citation.cfm?id=1251254.1251274

[17] Zhenmin Li and Yuanyuan Zhou. 2005. PR-Miner: Automatically Extracting Implicit Programming Rules and Detecting Violations in Large Software Code. SIGSOFT Softw. Eng. Notes 30, 5 (Sept. 2005), 306-315. https://doi.org/10.1145/ 1095430.1081755

[18] Jean loup Gailly and Mark Adler. 2018. The gzip home page. http://www.gzip.org/.

[19] Daniel Miessler. 2017. A grep Tutorial and Primer https://danielmiessler.com/study/grep/.
[20] Jeremy W. Nimmer and Michael D. Ernst. 2002. Automatic Generation of Program Specifications. SIGSOFT Softw. Eng. Notes 27, 4 (July 2002), 229-239. https: //doi.org/10.1145/566171.566213

[21] Jeremy W. Nimmer and Michael D. Ernst. 2002. Invariant Inference for Static Checking: An Empirical Evaluation. SIGSOFT Softw. Eng. Notes 27, 6 (Nov. 2002), 11-20. https://doi.org/10.1145/605466.605469

[22] Jeff H. Perkins and Michael D. Ernst. 2004. Efficient Incremental Algorithms for Dynamic Detection of Likely Invariants. SIGSOFT Softw. Eng. Notes 29, 6 (Oct. 2004), 23-32. https://doi.org/10.1145/1041685.1029901

[23] James Ralston. 2008. Red Hat Bugzilla âĂŞ Bug 438076. https://bugzilla.redhat.com/show bug.cgi?id $=438076$.

[24] Pang-Ning Tan, Michael Steinbach, Anuj Karpatne, and Vipin Kumar. 2005. Introduction to Data Mining: Chapter 6.

[25] Suresh Thummalapenta and Tao Xie. 2009. Mining Exception-handling Rules As Sequence Association Rules. In Proceedings of the 31st International Conference on Software Engineering (ICSE '09). IEEE Computer Society, Washington, DC, USA, 496-506. https://doi.org/10.1109/ICSE.2009.5070548

[26] V. Tiwari, V. Tiwari, S. Gupta, and R. Tiwari. 2010. Association rule mining: A graph based approach for mining frequent itemsets. In 2010 International Conference on Networking and Information Technology. 309-313. https://doi.org/ 10.1109/ICNIT.2010.5508505

[27] Mohammed J. Zaki. 2001. SPADE: An Efficient Algorithm for Mining Frequent Sequences. Mach. Learn. 42, 1/2 (Jan. 2001), 31-60. https://doi.org/10.1023/A: 1007652502315

[28] Mohammed J. Zaki and Ching-Jui Hsiao. 1999. CHARM: An Efficient Algorithm for Closed Association Rule Mining. Technical Report. COMPUTER SCIENCE, RENSSELAER POLYTECHNIC INSTITUTE. 